\title{
Ancient haplotypes of the HLA Class II region
}

\author{
Christopher K. Raymond, ${ }^{1,2}$ Arnold Kas, ${ }^{1}$ Marcia Paddock, ${ }^{1}$ Ruolan Qiu, ${ }^{1}$ Yang Zhou, ${ }^{1}$ \\ Sandhya Subramanian, ${ }^{1}$ Jean Chang, ${ }^{1}$ Anthony Palmieri, ${ }^{1,2}$ Eric Haugen, ${ }^{1}$ \\ Rajinder Kaul, ${ }^{1}$ and Maynard V. Olson ${ }^{1,3}$ \\ ${ }^{1}$ University of Washington Genome Center, Department of Medicine, University of Washington, Seattle, Washington 98195, USA
}

\begin{abstract}
Allelic variation in codons that specify amino acids that line the peptide-binding pockets of HLA's Class II antigen-presenting proteins is superimposed on strikingly few deeply diverged haplotypes. These haplotypes appear to have been evolving almost independently for tens of millions of years. By complete resequencing of 20 haplotypes across the $\sim 100-\mathrm{kbp}$ region that spans the HLA-DQA1, $-D Q B 1$, and -DRB1 genes, we provide a detailed view of the way in which the genome structure at this locus has been shaped by the interplay of selection, gene-gene interaction, and recombination.
\end{abstract}

[Supplemental material is available online at www.genome.org. The sequence data from this study have been submitted to GenBank under accession nos. AY663393-AY663415.]

Tens to hundreds of functionally distinct alleles have been described for the genes encoding the antigen-presenting molecules HLA-DQA1, $-D Q B 1$, and -DRB1 in the class II region of HLA (Bontrop et al. 1999; Marsh et al. 2000). Although there is detailed knowledge of coding-region variation in these genes, the data on haplotype evolution are fragmentary. Such information will be essential for understanding the molecular evolution of HLA and also for carrying out fine-structure mapping of associations between HLA haplotypes and the many human diseases that are influenced by an individual's HLA genotype (Price et al. 1999). Existing data on noncoding variation are either too anecdotal or too localized to particular gene segments to provide an overview of haplotype variation across any major segment of HLA. The few long-range studies that are available are limited to pairwise comparisons of arbitrarily chosen haplotypes (Guillaudeux et al. 1998; Horton et al. 1998; Gaudieri et al. 2000; Satta and Takahata 2000; Stewart et al. 2004); in contrast, short-range studies examine multiple haplotypes only in the immediate vicinity of particular polymorphic exons (McGinnis et al. 1994; Bergstrom et al. 1998; Kotsch and Blasczyk 2000). Despite their limited scope, these data clearly reveal patterns and levels of haplotype variation that have not been seen elsewhere in the human genome (International SNP Map Working Group 2001). At the high end, pairwise divergences reach $5 \%-10 \%$ across tens of thousands of base pairs, while at the low end they collapse to background levels of $0.1 \%$ or less.

The lack of systematic, long-range sequences of many haplotypes is largely due to the technical difficulty of acquiring haplotype-resolved sequences across tens of thousands of base pairs from the genomes of many individuals. For our analysis, we developed an efficient fosmid-based recloning system to obtain the sequences of 20 human haplotypes, as well as single sequences from a chimpanzee and a gorilla, across an average of $106 \mathrm{kbp}$ of the class II region. Most of the human sequences, which were obtained from a geographically diverse panel of 10 individuals,

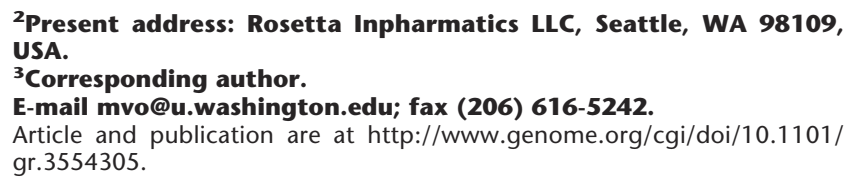

include all of the $D Q B 1, D Q A 1$, and $D R B 1$ genes. We believe that these data represent the first haplotype-resolved resequencing study of this breadth and depth for any locus in any organism.

\section{Results}

We based our analysis of the $D Q B 1-D Q A 1-D R B 1$ region on a multiple alignment (Supplemental Table 1) of 23 sequences: one chimpanzee haplotype, one composite gorilla haplotype, and 21 human haplotypes (Fig. 1). Of the 21 human haplotypes, 20 were from our study and one was from the Human Genome Project. Multiple alignment of the 23 sequences led to 59,668 fully aligned sites, 5328 of which have at least two human alleles. Considering only the human-human comparisons, maximal pairwise divergences range from $2.1 \%$ to $9.3 \%$, whereas minimal divergences are everywhere $<0.1 \%$ (Fig. 2). Human-chimpanzee, human-gorilla, and chimpanzee-gorilla divergences have essentially the same upper limit as the human-human comparisons, but the lower limit for the inter-species comparisons is $\sim 1 \%$. Indeed, over most of the region studied, there are examples of chimpanzee-human or gorilla-human comparisons that are in the $1 \%-2 \%$ range. Since this value is similar to genome-wide averages for interspecies comparisons among humans, chimpanzees, and gorillas (Chen and Li 2001), there is no indication of an unusually high mutation rate in the class II region.

We interpret the exceptionally high divergences between some pairs of haplotypes, regardless of the species from which they were sampled, as being due to a long history of independent haplotype evolution. This model, which requires extensive sequence variation to have flowed through many speciation bottlenecks, is well established for the coding-region polymorphisms of both Class I and Class II HLA genes (Lawlor et al. 1988; Mayer et al. 1988; Fan et al. 1989; Gyllensten and Erlich 1989; Gyllensten et al. 1990). Our data extend this evolutionary model to the entire class II region. Because there are no known functional elements interspersed among the $H L A-D Q B 1,-D Q A 1$, and -DRB1 genes (Beck and Trowsdale 1999; The MHC Sequencing Consortium 1999), we presume that most noncoding sequences have evolved neutrally, while balancing selection has acted on the functionally important variation within the three highly poly- 


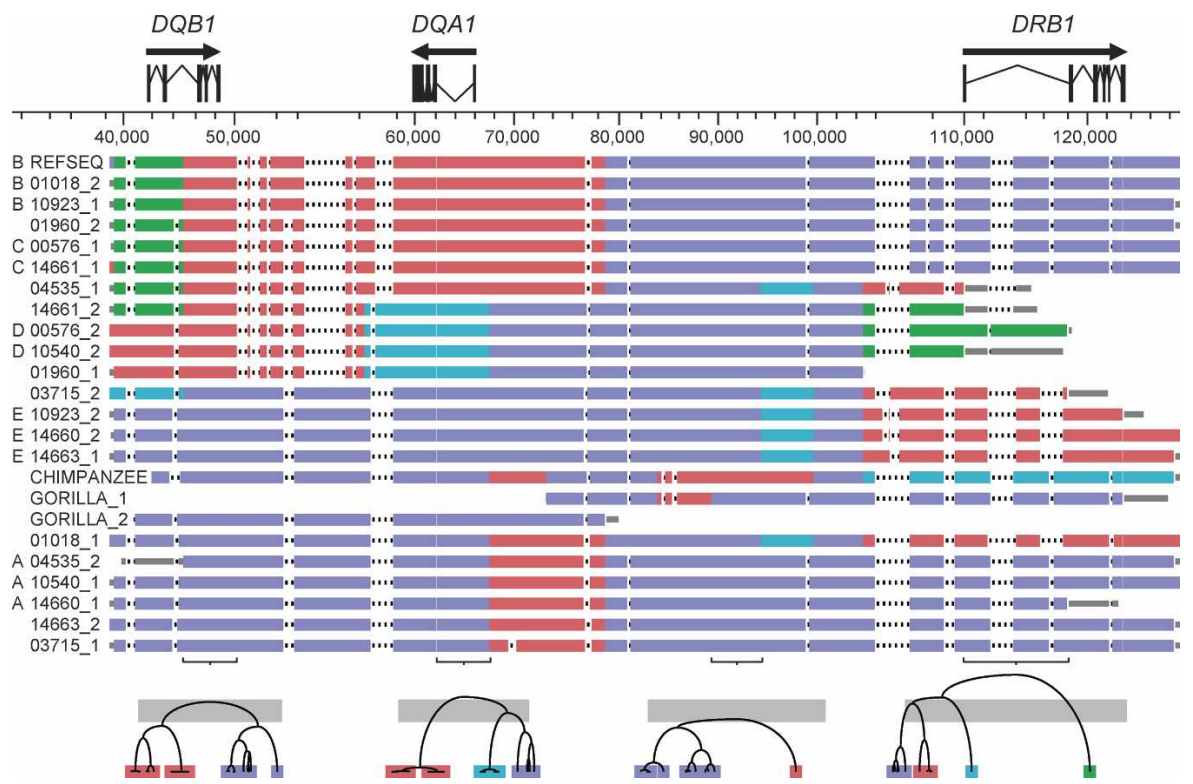

Figure 1. A visual representation of the multi-aligned haplotypes. The arrows over the gene models indicate the direction of transcription. Haplotype designations are indicated on the left, including membership in haplotype groups A, B, C, D, or E, when appropriate. Haplotypes within a group are nearly identical across the entire region. The coordinate system, in base pairs, is based on haplotype RefSeq (see Methods). Physical distance in the figure corresponds to the longest path through the multi-alignment; deletions relative to the longest path are indicated by gaps in the appropriate haplotypes (and, if space allows, by a series of dots). However, the tick marks and coordinates on the horizontal axis refer to RefSeq coordinates. Coloring of the haplotypes clusters them locally according to the deep branches of the phylogenetic tree to which they belong. The local clusterings were achieved by constructing molecular phylogenies for nonoverlapping 5-kbp windows across the region. Sample phylogenetic trees are shown at the bottom of the figure. The bottoms of the horizontal gray bars across the trees, which correspond to a pairwise-sequence divergence of $4 \%$, indicate the arbitrary threshold for assigning a group of haplotypes the same color in a local region. The gray bars visible at the ends of most haplotypes are segments that could not be assigned a color in that region because they ended within one of the 5-kbp windows used for tree construction. The gorilla sequence, although treated in most instances as a single composite haplotype, is shown here as two overlapping sequences, GORILLA_1 and GORILLA_2, because these two sequences, which are true haplotypes from a single individual, are of different parental origins.

morphic genes (Hughes and Nei 1989; Begovich et al. 1992). Assuming normal mutation rates and neutrality at most noncoding sites, the most dissimilar haplotypes would have had to have evolved independently for $\sim 40$ million years (Myr) to have diverged by as much as 9\% (Satta et al. 1999).

To produce the current pattern of haplotype divergence, recombination between highly divergent haplotypes must have been strongly suppressed relative to the genome-wide average recombination rate. Although "hitchhiking" of neutral variation on selected sites is a well established phenomenon (Smith and Haigh 1974; Kaplan et al. 1989), the level of recombinational suppression required to maintain long-range haplotype integrity over $40 \mathrm{Myr}$ is extraordinary. In family studies, the sex-averaged recombination rate on chromosome 6 in the HLA region is $\sim 0.5$ cM/Mbp (Martin et al. 1995; Kong et al. 2002), a value similar to the genome-wide average. At this recombination rate, the interval over which there is a probability of 0.5 that there will have been no recombination in $40 \mathrm{Myr}$ is $<100 \mathrm{bp}$. This estimate varies inversely with elapsed time and recombination rate and directly with the assumed generation time. Although none of these parameters is well established, any plausible set of values predicts that hitchhiking should be limited to a few hundred base pairs or less. In contrast to this prediction, we observe strong linkage disequilibrium across most of the multi-aligned region, a dis- tance of tens of thousands of base pairs (Fig. 3). This result, which is consistent with previous studies based on limited sets of genetic markers (Begovich et al. 1992; Sanchez-Mazas et al. 2000; Stenzel et al. 2004), indicates that evolutionarily successful recombination events between deeply diverged haplotypes in most subintervals of the class II region have been rare relative to expectations based on genome-wide recombination rates.

Nonetheless, a few clear instances of such events are evident when particular pairs of haplotypes are compared. We discovered these examples by examining all 210 distinct pairwise comparisons that can be made among the 21 human haplotypes we analyzed. This analysis was simplified by the observation that 13 of the 21 haplotypes fall into five groups, designated A-E, which are defined by near identity within each group (i.e., intragroup divergences, averaged across the $D Q B 1-D Q A 1-D R B 1$ region, are $<0.3 \%)$. We found one particularly dramatic example of what appears to have been a simple, recent reciprocal-recombination event: haplotype 04535_1 is nearly identical to the group $\mathrm{E}$ haplotypes over half the region and to the group $\mathrm{C}$ haplotypes over the remainder (Fig. 4B,C). In contrast, the group $\mathrm{C}$ and $\mathrm{E}$ haplotypes are deeply divergent across nearly the whole multialigned region (Fig. 4A). This pattern is suggestive of a reciprocal recombination between a C- and an E-group haplotype that occurred very recently compared to the time over which these two haplotypes have been diverging. Because this recent event affected the structure of only one of the 21 human haplotypes, it did not have a major influence on disequilibrium values for SNPs on opposite sides of the recombination junction. A more complex example is evident in pairwise comparisons between Band D-group haplotypes, which are highly divergent except in two regions: one is between the $D Q B 1$ and $D Q A 1$ genes, and the other is in a 20-kbp interval between DQA1 and DRB1 (Fig. 4D). The latter feature appears to reflect either two successive recent recombination events or a very long gene-conversion track. Unlike the situation for haplotype 04535_1, we did not observe reciprocal divergence patterns at either of the apparent recombination sites. Two other recombinant haplotypes are evident in Figure 1 (e.g., 14661_2 appears to be a C/D recombinant and 01018_1 appears to be an A/E recombinant); however, neither of these examples is nearly as dramatic as those displayed in Figure 4 , since there is substantial divergence between one or both segments in the recombinant haplotypes and the closest match in our data set.

The strong dip in the divergence of the B- and D-group haplotypes between the $D Q B 1$ and $D Q A 1$ genes (Fig. 4D) is of special interest. We explored the relationship between all the haplotypes in this region by constructing molecular phylogenies 


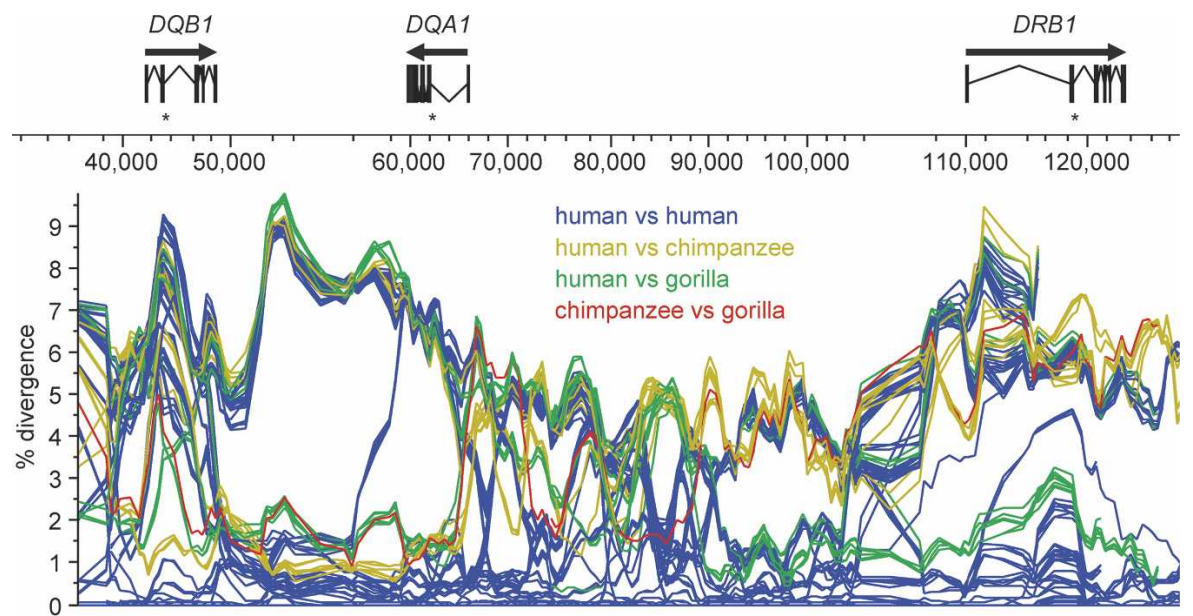

Figure 2. Pairwise comparisons among the haplotypes. A separate line is plotted for each pairwise comparison, but only the different classes of interspecies comparison are assigned different colors. The coordinate system is the same as that in Figure 1. The vertical axis indicates percent divergence between the pairs of haplotype sequences. Pairwise divergences were calculated as averages for 2-kbp windows that slid 500 base pairs between points. The arrows over the gene models indicate the direction of transcription. The most variable exons of the three genes (in all cases, exon 2) are indicated by an asterisk.

from the data in a series of 2-kbp windows that tiled the DQB1$D Q A 1$ interval. The most striking feature of such phylogenies is the persistence of at least two deeply diverged branches throughout the12.3-kbp intergenic region (Fig. 5A-D). Additional deep splits occur in regions adjacent to the $D Q B 1$ and $D Q A 1$ genes. If recent recombination events had occurred between haplotypes belonging to the two deep intergenic branches, there would be sudden, major shifts in the topology of successive trees. We found no such examples in our data set. Even within each of the two major branches, tree topology is generally conserved, particularly in the branch of Figure 5, which includes the B-, C-, and D-group haplotypes. The only obvious example of a recent recombination event within this branch involves haplotype 14661_2, which appears to have been created by recent recombination between a C- and a D-group haplotype: haplotype 14661_2 clusters with the C-group haplotypes at the DQB1 end of the interval (Fig. 5A,B) and the D-group haplotypes at the DQA1 end (Fig. 5C,D).

The collapse in the depth of both major sublineages of the bifurcated tree in the DQB1-DQA1 interval (Fig. 5A-D) poses something of a dilemma. Low intrabranch divergence in this region relative to surrounding regions must be due to local changes in the mutation rate, recombination rate, or strength of selection. A regionally low mutation rate is implausible since interbranch divergence in this region is high and the divergences of human haplotypes from their closest chimpanzee and gorilla counterparts are unremarkable. Action of purifying selection within each branch is also implausible, since there are no known functional elements in the intergenic region, and, even if such elements exist, most SNPs are still likely to be evolving neutrally. We presume that the collapse of intrabranch diversity in the $D Q B 1-D Q A 1$ region is due to a subtle interplay between recombination and selection. In this model, recombination occurs at rates high enough to shorten intrabranch coalescence times-by decreasing hitchhiking of neutral alleles on sites within the $D Q B 1$ and $D Q A 1$ genes that are under balancing selection-but low enough to preserve key aspects of tree topology.

We found a simple correlation between noncoding haplo- types and coding-region alleles, particularly in the $D Q B 1-D Q A 1$ region. This correlation is evident in Figure 6, in which classical DQB1-DQA1-DRB1 allele designations are juxtaposed with a molecular phylogeny based on a typical segment of the $D Q B 1-D Q A 1$ intergenic region. This figure is easily interpreted because the allele designations, like the tree itself, are based on a hierarchical classification system (e.g., all classical ${ }^{*} 03$ alleles have fewer sequence differences from one another than with any ${ }^{*} 02$ alleles, and all *0301 alleles have fewer differences from each other than with any ${ }^{*} 0302$ alleles). The nearly perfect correlation between the phylogenetic tree built from the complete haplotype sequences and the hierarchical clustering of the functional alleles of $D Q B 1$ and $D Q A 1$ reflects the nearly complete linkage disequilibrium between neutral and selected sites in this region. Indeed, the two deep branches of the intergenic trees (Fig. 5B,C) correlate absolutely with the DQ haplotypes observed in diverse human populations. In numerous large studies of multiple populations, $D Q B 1{ }^{*} 02$ and ${ }^{*} 03$ alleles (bottom branch in Fig. 5B,C) are not found on the same haplotypes as $D Q A 1{ }^{*} 01$ alleles (top branch in Fig. 5B,C); similarly, $D Q B 1{ }^{*} 05$ and ${ }^{*} 06$ alleles (top branch) are not found on the same haplotypes as $D Q A 1{ }^{*} 03$ and ${ }^{*} 05$ alleles (bottom branch) (Imanishi et al. 1992; Charron 1997; Begovich et al. 2000). Hence, the absence of detectable historical recombination between the two deeply diverged branches simply reflects the rules governing "forbidden" combinations of $D Q B 1$ and $D Q A 1$ alleles, which encode the two subunits of the heterodimeric DQ protein. This result is particularly satisfying since there is a well established biochemical basis for this "gene-gene interaction:" the protein products of forbidden allele combinations have been shown to form unstable heterodimers in vitro (Kwok et al. 1993). Therefore, selection against recombinants-rather than an absence of recombination events-may account for the topology of the haplotype trees in the $D Q B 1-D Q A 1$ region. However, it should be noted that putative exceptions to the usual rules governing $D Q B 1-D Q A 1$ associations have been reported to exist at significant frequencies in some populations (Grahovac et al. 1998). If validated, we predict that the haplotypes that account for these exceptions will reflect relatively recent recombi-

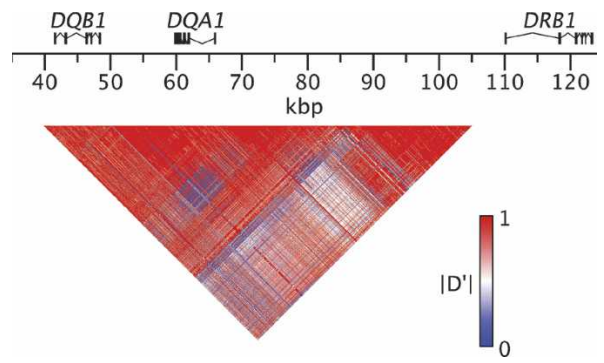

Figure 3. Pairwise linkage disequilibrium between SNPs in the $D Q B 1$ $D Q A 1-D R B 1$ region. The matrix display shows linkage disequilibrium between pairs of SNPs, measured by the statistic $\left|D^{\prime}\right|$ (see Methods). The coordinates correspond to those defined in the Figure 1 legend.

\section{Genome Research}

www.genome.org 


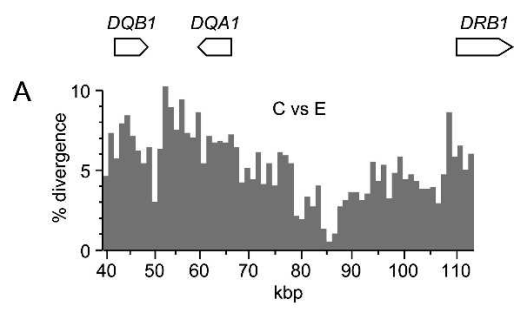

B

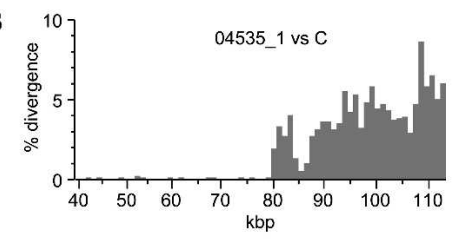

C
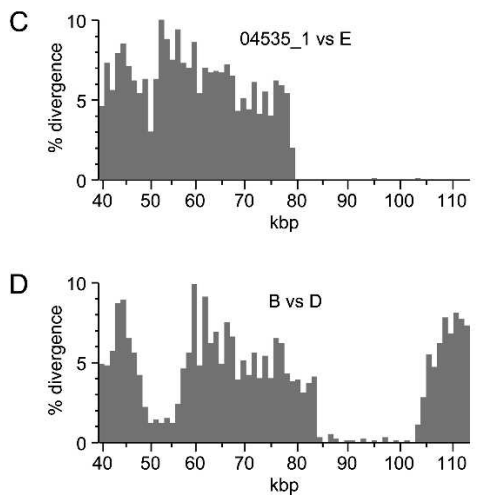

Figure 4. Histograms of the pairwise divergence among selected haplotypes. The coordinate system is the same as that employed in Figure 1. Divergences were calculated for 1-kbp bins, based on RefSeq coordinates, counting only discrepancies at nucleotide positions that could be fully aligned with all other haplotypes for which sequence was available in a given region. (A) Representative C-group haplotype compared to a representative E-group haplotype. (B) Recombinant haplotype 04535_1 compared to a representative C-group haplotype. (C) Recombinant haplotype 04535 1 compared to a representative E-group haplotype. $(D) \mathrm{A}$ representative B-group haplotype compared to a representative D-group haplotype. The actual haplotypes used to represent haplotype groups $B$, C, D, and E (see legend to Fig. 1) were 01018_2, 00576_1, 00576_2, and 10923_2, respectively.

nation between haplotypes associated with the two deep branches that characterize the $D Q B 1-D Q A 1$ intergenic region.

The situation at the DRB1 end of the class II region is more complex. Substantial linkage disequilibrium exists between the strong haplotypes in the $D Q B 1-D Q A 1$ region and the classical $D R B 1$ alleles. However, unlike the situation between DQB1 and $D Q A 1$, where "forbidden" combinations of alleles are on branches of the molecular phylogeny that remain deeply diverged across the whole $D Q B 1-D Q A 1$ interval, there is no consistent relationship between the extent of haplotype divergence between $D Q A 1$ and $D R B 1$ and the extent to which the classical $D Q A 1$ and DRB1 alleles on these haplotypes are in linkage disequilibrium. Nonetheless, examples of this phenomenon do exist and are likely to reflect the effects of unusually longstanding or strong selection for maintenance of certain preferred combinations of $D Q B 1, D Q A 1$, and DRB1 alleles. The clearest example involves the $\mathrm{C}$ - and E-group haplotypes, which are deeply divergent across nearly the entire class II region (Fig. 4A). The C-group haplotypes carry DQB1-DQA1-DRB1 alleles *030-*050*-*110, whereas the E-group haplotypes carry alleles ${ }^{*} 060-{ }^{*} 010-{ }^{*} 150$. In one survey of over 2000 individuals from a worldwide sampling of 18 human populations, haplotypes with the C- and E-groups' combination of DQB1, DQA1, and DRB1 alleles were found in nearly all populations at frequencies ranging from a few percent to over $20 \%$ (Imanishi et al. 1992). In contrast, the allele combination ${ }^{*} 03-{ }^{*} 05-^{*} 150$ present on the C-E-recombinant haplotype, 04535_1 (Fig. 4B,C), was not found in this large study. Interestingly, the allele combination that would be associated with the reciprocal C-E recombinant, ${ }^{*} 060-{ }^{*} 010-{ }^{*} 110$, was found at a frequency of $9 \%$ in a South African population, and at a lower frequency among American blacks (Imanishi et al. 1992).

\section{Discussion}

By analyzing 21 human haplotypes across the HLA class II region, the sequences of 20 of which were newly acquired for this study, we have demonstrated a dramatic pattern of haplotype divergence that appears to extend back $\sim 40 \mathrm{Myr}$. Although we found some examples of recent recombination events between deeply diverged haplotypes, the overall pattern of linkage disequilibrium across the region requires that such events be infrequent relative to predictions based on genome-wide recombination rates. There was no indication of scrambling of functional alleles at the class II genes, $D Q B 1, D Q A 1$, and DRB1, across highly divergent haplotypes by gene conversion or multiplerecombination events combined with selection. Overall, the data are largely consistent with a model in which deeply diverged haplotypes have evolved independently for tens of millions of years.

This model could either reflect infrequent recombination or selection against recombinant haplotypes. Despite longstanding interest in distinguishing between these two models (Cullen et al. 1997) - and evidence in an adjacent, much less polymorphic, segment of the HLA class II region that recombination hot spots can be the dominant influence on linkage disequilibrium patterns (Kauppi et al. 2003) - there is presently no basis for determining their relative contributions in the DQB1-DQA1-DRB1 region. The data presented here suggest a clear path forward for resolving this long-standing debate. High-resolution recombination studies, based on sperm typing, must be carried out on carefully selected men. The essential question is whether or not recombination rates are strongly suppressed in regions of heterozygosity for high haplotype divergence. Previous studies (Cullen et al. 2002), whether based on sperm typing or pedigree analysis, have not been able to control for this critical variable because of a lack of reference data on the common haplotype sequences present in the DQB1-DQA1-DRB1 region.

Recent long-range sequencing data for two HLA haplotypes (Stewart et al. 2004) allow us to put the extraordinary sequence polymorphism we observe in the class II region into a broader context. The two available sequences-from the COX and PGF cell lines, both of which are homozygous across the HLA locus due to identity-by-descent-fit cleanly into our B (COX) and E (PGF) haplotype groups. The region we analyzed captures most of the interval of extreme divergence $(>2 \%)$ between the COX and PGF haplotypes. The overall pattern of divergence between the COX and PGF haplotypes across all of HLA is one of peaks of unusual divergence at sites of highly polymorphic genes (HLA-A, the $H L A-B /-C$ region, the $H L A-D Q B 1-D R B 1-D Q B 1$ region ana- 

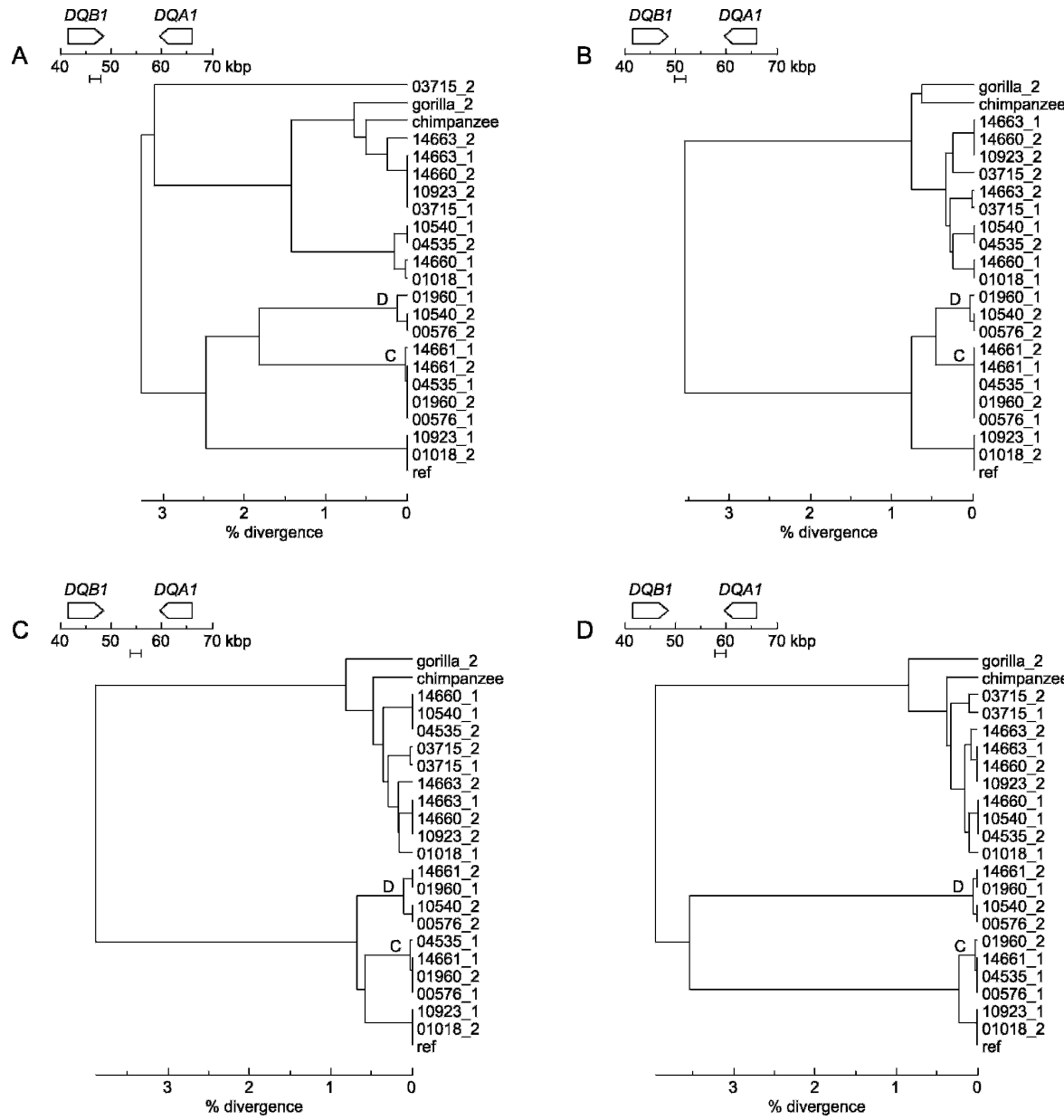

Figure 5. Molecular phylogenies for the 23 haplotypes in four 2-kbp windows sampled from the $D Q B 1-D Q A 1$ interval. The coordinate system is the same as that employed in Figure 1. Parts $(A-D)$ differ only in the position of the 2-kbp window that was analyzed: horizontal bars below the coordinate axes indicate the position of the window that was the source of data for each tree. Branches leading to Cand D-group haplotypes (see Fig. 1 legend) are labeled.

lyzed here, and HLA-DPB1), separated by normal or near-normal levels of variation; the typical extent of highly divergent $(>0.5 \%)$ regions, of which the DQB1-DQA1-DRB1 gene cluster is the most dramatic example, is $100-300-k b p$.

We hypothesize that genomic regions of the type described here will occur commonly in biology even if extreme examples are rare in any given genome. The prerequisites are a cluster of genes that are individually under balancing selection and whose products interact. Under these circumstances, theory predicts precisely the type of long-range hitchhiking of neutral alleles on selected sites that we observe in the HLA class II region (Kelly and Wade 2000). Other gene clusters that are likely to exhibit similar effects include those that encode key components of the selfincompatibility systems present in many flowering plants (Charlesworth et al. 2003; Franklin-Tong and Franklin 2003; Hiscock and Tabah 2003).

Although new data will be needed to resolve the relative contributions of a low recombination rate and selection for preferred combinations of alleles to the evolution of the class II region, we hypothesize that both mechanisms are important. Certainly, it is plausible from data on model organisms that recombination rates might drop significantly when allelic seg- ments have diverged by as much as $5 \%$ 10\% (Shen and Huang 1989). However, we consider it likely that selection for preferred combinations of alleles is the decisive determinant of haplotype divergence in the class II region. This view suggests that haplotype divergence is predominantly the effect rather than the cause of the prevailing pattern of allelic association.

In addition to being important in its own right, the class II region provides a model for the types of genomic footprints that are left by the interplay of recombination, balancing selection, and gene-gene interaction. In the case of the HLA class II gene cluster, the footprint is uniquely strong and readily detectable without recourse to sophisticated statistical tests. However, it may prove possible to detect comparable, albeit weaker, footprints when similar processes have acted over shorter time intervals. The methods we employed provide a potentially general path toward the detailed analysis of genome segments with these properties.

\section{Methods}

\section{DNA samples}

DNA samples were obtained from the Coriell Cell Repositories. Self-descriptions of the nationality or geographic ancestry of the anonymous human donors are included here. Even though these descriptors do not conform to a consistent standard, they do indicate that the panel provides a diverse, worldwide sampling of human genetic variation: 04535 (Asian, Japanese), 10540 (Melanesian), 14660 (Black, African American), 01018 (Caucasian, Puerto Rican), 10923 (Caucasian, German), 00576 (Asian, Chinese), 14661 (Black, African American), 14663 (Black, African American), 01960 (Caucasian, Mexican), 03715 (Caucasian, European). Catalog numbers for chimpanzee and gorilla samples were 03646 and 05251, respectively.

\section{Fosmid cloning}

Fosmid libraries (Kim et al. 1992) were prepared by cloning 30$50-\mathrm{kbp}$ fragments of genomic DNA, prepared by partial digestion with Sau3AI (New England Biolabs), and size-fractionated by preparative agarose gel electrophoresis into the BamH1 site of standard fosmid vectors (most commonly, pCC1FOS from Epicentre). Packaging into infectious bacteriophage lambda particles was carried out with Gigapack III XL lambda-packaging extract (Stratagene). The clones were propagated in XL1-Blue MR E. coli cells. After titering of the packaged ligation mixtures, pools of $\sim 3000-5000$ clones were created by infection of an E. coli culture, which was then grown overnight under chloramphenicol selection $(12 \mu \mathrm{g} / \mathrm{mL})$. One aliquot of the cultures was stored for later clone isolation, and another aliquot was used to prepare DNA samples for PCR analysis with a set of PCR assays, spaced at in-

\section{Genome Research}

www.genome.org 


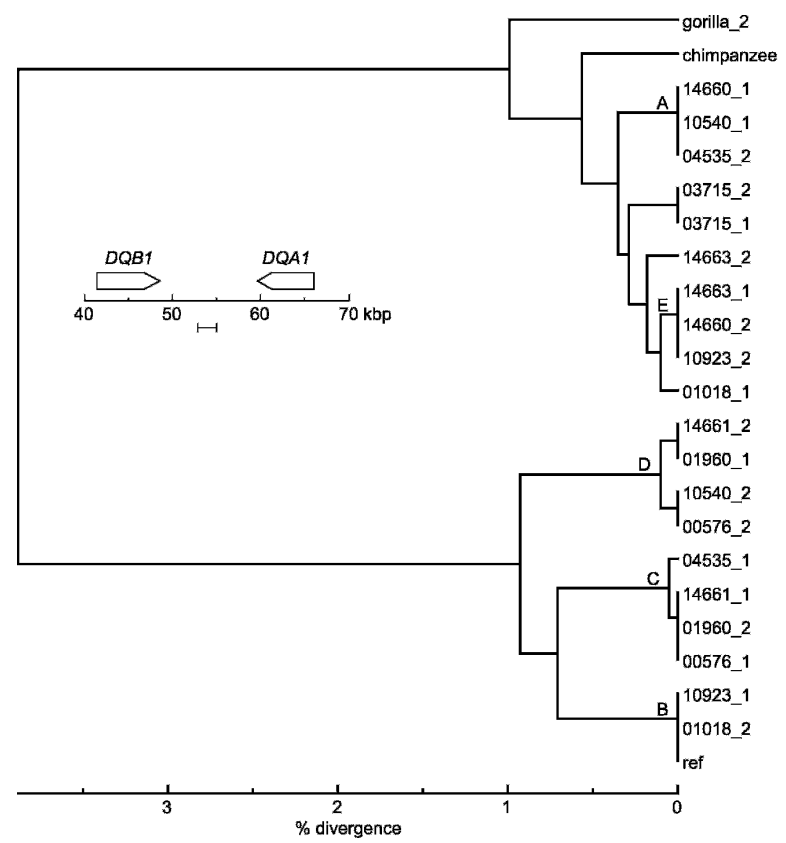

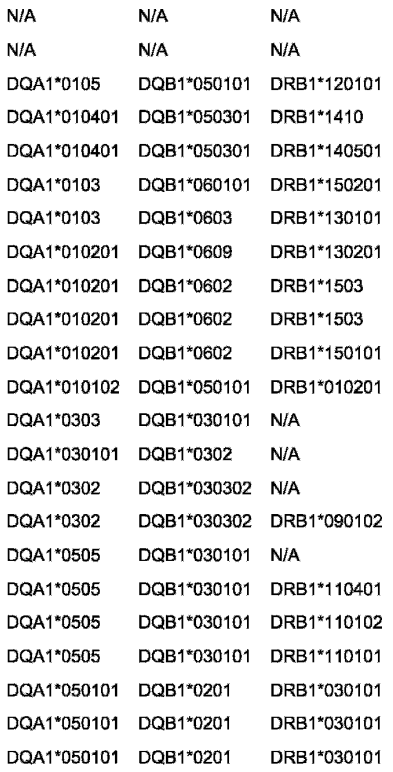

Figure 6. Classical $D Q A 1, D Q B 1$, and $D R B 1$ alleles present on the 21 human haplotypes juxtaposed to a molecular phylogeny from the middle of the $D Q B 1-D Q A 1$ intergenic region. The phylogenetic tree is redrawn from Figure $5 \mathrm{C}$ with branches leading to haplotype groups $\mathrm{A}-\mathrm{E}$, as defined in the Figure 1 legend, labeled. Entries are marked N/A ("not available") either because there is not a match to an established human allele — as was true for the gorilla and chimpanzee alleles —or insufficient data were available to establish a match.

tervals of $\sim 10 \mathrm{kbp}$ across the region analyzed. A list of these assays is included in Supplemental Table 2. Because of the high polymorphism across the region, allelic variation was present in most amplicons for most individuals. The allelic variant present in a subset of the amplicons, as amplified from particular fosmid pools, was determined by sequencing the PCR products. Haplotype-specific-STS-content maps (Green and Olson 1990) of the target region were constructed from the PCR data. Individual fosmid clones spanning each haplotype were isolated from an appropriately chosen set of positive pools. The clone-isolation procedure involved one or two cycles of subpool creation followed by plating for single colonies.

\section{DNA sequencing}

Isolated fosmids were sequenced by standard shotgun-sequencing procedures with data collection on ABI 3700 capillary sequencers. Assembly was with the phrap assembler (http://www .phrap.org/). Finishing relied on targeted data acquisition designed by the Autofinish program (Gordon et al. 2001), manual assessment of residual uncertainties with the aid of the Consed program (Gordon et al. 1998), and resolution of these problem regions through custom-designed experiments. Haplotype sequences were constructed by melding the overlapping set of fosmid sequences obtained from each haplotype.

\section{Reference sequence}

The reference sequence of the target region, RefSeq, was based on the July 2003 assembly of the human genome sequence; position 1 in RefSeq corresponds to chromosome 6 coordinate $32,722,342$; RefSeq is on the opposite strand relative to the database entry (http://genome.ucsc.edu/).

\section{Sequence alignments}

Pairwise and multiple alignments of the haplotypes were carried out using a program developed during the present study. Pairwise alignments of each haplotype with RefSeq were constructed using an algorithm that finds a maximal chain of perfect matches of 20 or more nucleotides. The algorithm then fills in intervening regions by using a standard dynamic programming method (Durbin et al. 1998). The multiple alignment was based on the set of pairwise alignments with RefSeq. Nucleotide positions were only included in the multiple alignment when they could be uniquely aligned across all haplotypes. The entire multiple alignment is included in Supplemental Table 1.

\section{Linkage disequilibrium analysis}

The data displayed in Figure 3 are based on the statistic $\left|\mathrm{D}^{\prime}\right|$, which ranges from 0 (random association between two SNPs across the available haplotypes) to 1 (maximum possible positive or negative association, considering the allele frequencies of the two SNPs) (Lewontin 1964). The analysis was only carried out across the region for which we had data on all 21 human haplotypes, and it only included positions at which all 21 haplotypes could be aligned. Furthermore, we excluded SNPs in which the minor allele occurred two or fewer times, and we also excluded SNPs that are likely to have arisen by mutations at CG dinucleotides. Specifically, SNPs were excluded if they belonged to a dinucleotide in which two out of the three sequences CG, TG, and CA were represented among the 21 haplotypes. The filtering out of rare dinucleotides increases the sensitivity of $\left|\mathrm{D}^{\prime}\right|$ to the effects of historical recombination events, and the elimination of most CGrelated mutations decreases noise due to recurrent mutation. For the display in Figure 3, each of the 3468 SNPs that survived the filtering was assigned a range of coordinates that includes half the distance (in REFSEQ coordinates) to each of the SNPs that bracketed it. The details of this procedure, which produces a linear coordinate system at the expense of giving different SNPs different weights in the display, affect only the finest-scaled of the visible features of the figure.

\section{Phylogenetic trees}

The phylogenetic trees in Figures 1 and 5 were constructed using the Phylip package, version 3.5 (http://evolution.genetics. washington.edu/phylip.html), using the neighbor-joining/ UPGMA (unweighted pair group methods with averages) method.

\section{Allele assignments}

Classical HLA alleles were assigned to the human haplotypes by comparing the $H L A-D Q B 1,-D Q A 1$, and $-D R B 1$ coding regions with data tabulated in the April 2004 update of the Anthony Nolan database (http://www.anthonynolan.org.uk/HIG/data .html). In most instances, allele assignments were based on exact matches at all diagnostic nucleotide positions. Exceptions are enumerated in Supplemental Table 3. 


\section{Database submissions}

The 20 human, the chimpanzee, and the two overlapping gorilla haplotypes sequenced for this study have been deposited in GenBank with accession nos. AY663393-AY663415.

\section{Acknowledgments}

We thank D. Geraghty and A. Lernmark for discussions that stimulated our interest in genetic variation at HLA. In addition to the cited authors, numerous members of the staff of the University of Washington Genome Center contributed to the collection and management of the data. This work was supported by Center of Excellence in Genome Science grant P50 HG02351 from the NIH National Human Genome Research Institute.

\section{References}

Beck, S. and Trowsdale, J. 1999. Sequence organisation of the class II region of the human MHC. Immunol. Rev. 167: 201-210.

Begovich, A., McClure, G.R., Suraj, V.C., Helmuth, R.C., Fildes, N., Bugawan, T.L., Erlich, H.A., and Klitz, W. 1992. Polymorphism, recombination, and linkage disequilibrium within the HLA class II region. J. Immunol. 148: 249-258.

Begovich, A.B., Klitz, W., Steiner, L.L., Grams, S., Suraj-Baker, V., Hollenbach, J., Trachtenberg, E., Louie, L., Zimmerman, P.A., Hill, A.V.S., et al. 2000. HLA-DQ haplotypes in 15 different populations. In Major histocompatibility complex: Evolution, structure, and function (ed. M. Kasahara), pp. 412-426. Springer-Verlag, Tokyo.

Bergström, T.F., Josefsson, A., Erlich, H.A., and Gyllensten, U. 1998 Recent origin of HLA-DRB1 alleles and implications for human evolution. Nat. Genet. 18: 237-242.

Bontrop, R.E., Otting, N., de Groot, N.G., and Doxiadis, G.G.M. 1999. Major histocompatibility complex class II polymorphisms in primates. Immunol. Rev. 167: 339-350.

Charlesworth, D., Mable, B.K., Schierup, M.H., Bartolome, C., and Awadalla, P. 2003. Diversity and linkage of genes in the self-incompatibility gene family in Arabidopsis lyrata. Genetics 164: $1519-1535$.

Charron, D. 1997. In HLA: Genetic diversity of HLA functional and medical implication. EDK, Paris.

Chen, F.C. and Li, W.H. 2001. Genomic divergences between humans and other hominoids and the effective population size of the common ancestor of humans and chimpanzees. Am. J. Hum. Genet. 68: $444-456$

Cullen, M., Noble, J., Erlich, H., Thorpe, K., Beck, S., Klitz, W., Trowsdale, J., and Carrington, M. 1997. Characterization of recombination in the HLA Class II region. Am. J. Hum. Genet. 60: $397-407$

Cullen, M., Perfetto, S.P., Klitz, W., Nelson, G., and Carrington, M. 2002. High-resolution patterns of meiotic recombination across the human major histocompatibility complex. Am. J. Hum. Genet. 71: 759-776.

Durbin, R., Eddy, S., Krogh, A., and Mitchison, G. 1998. Biological sequence analysis: Probabilistic models of proteins and nucleic acids. Cambridge University Press, Cambridge, UK.

Fan, W.M., Kasahara, M., Gutknecht, J., Klein, D., Mayer, W.E., Jonker, M., and Klein, J. 1989. Shared class II MHC polymorphisms between humans and chimpanzees. Hum. Immunol. 26: 107-121.

Franklin-Tong, V.E. and Franklin, F.C. 2003. The different mechanisms of gametophytic self-incompatibility. Philos. Trans. R. Soc. Lond. B Biol. Sci. 358: 1025-1032.

Gaudieri, S., Dawkins, R.L., Habara, K., Kulski, J.K., and Gojobori, T. 2000. SNP profile within the human major histocompatibility complex reveals an extreme and interrupted level of nucleotide diversity. Genome Res. 10: 1579-1586.

Gordon, D., Abajian, C., and Green, P. 1998. Consed: A graphical tool for sequence finishing. Genome Res. 8: 195-202.

Gordon, D., Desmarais, C., and Green, P. 2001. Automated finishing with autofinish. Genome Res. 11: 614-625.

Grahovac, B., Sukernik, R.I., O’hUigin, C., Zaleska-Rutczynska, Z., Blagitko, N., Raldugina, O., Kosutic, T., Satta, Y., Figueroa, F., Takahata, N., et al. 1998. Polymorphism of the HLA class II loci in Siberian populations. Hum. Genet. 102: 27-43.

Green, E.D. and Olson, M.V. 1990. Chromosomal region of the cystic fibrosis gene in yeast artificial chromosomes: A model for human genome mapping. Science 250: 94-98.

Guillaudeux, T., Janer, M., Wong, G.K., Spies, T., and Geraghty, D.E. 1998. The complete genomic sequence of $424,015 \mathrm{bp}$ at the centromeric end of the HLA class I region: Gene content and polymorphism. Proc. Natl. Acad. Sci. 95: 9494-9499.

Gyllensten, U.B. and Erlich, H.A. 1989. Ancient roots for polymorphism at the HLA-DQ $\alpha$ locus in primates. Proc. Natl. Acad. Sci. 86: 9986-9990.

Gyllensten, U.B., Lashkari, D., and Erlich, H.A. 1990. Allelic diversification at the class II DQB locus of the mammalian major histocompatibility complex. Proc. Natl. Acad. Sci. 87: 1835-1839.

Hiscock, S.J. and Tabah, D.A. 2003. The different mechanisms of sporophytic self-incompatibility. Philos. Trans. R. Soc. Lond. B Biol. Sci. 358: 1037-1045.

Horton, R., Niblett, D., Milne, S., Palmer, S., Tubby, B., Trowsdale, J., and Beck, S. 1998. Large-scale sequence comparisons reveal unusually high levels of variation in the HLA-DQB1 locus in the class II region of the human MHC. J. Mol. Biol. 282: 71-97.

Hughes, A.L. and Nei, M. 1989. Nucleotide substitution at major histocompatibility complex class II loci: Evidence for overdominant selection. Proc. Natl. Acad. Sci. 86: 958-962.

Imanishi, T., Akaza, T., Kimura, A., Tokunaga, K., and Gojobori, T. 1992. Allele and haplotype frequencies for HLA and complement loci in various ethnic groups. In HLA 1991 (eds. K. Tsuji, et al.), Vol. I, pp.1065-1204. Oxford University Press, Oxford, UK.

International SNP Map Working Group 2001. A map of human sequence variation containing 1.42 million single nucleotide polymorphisms. Nature 409: 928-933.

Kaplan, N.L., Hudson, R.R., and Langley, C.H. 1989. The "hitchhiking effect" revisited. Genetics 123: 887-899.

Kauppi, L., Sajantila, A., and Jeffreys, A.J. 2003. Recombination hotspots rather than population history dominate linkage disequilibrium in the MHC class II region. Hum. Mol. Genet. 12: 33-40.

Kelly, J.K. and Wade, M.J. 2000. Molecular evolution near a two-locus balanced polymorphism. J. Theor. Biol. 204: 83-101.

Kim, U.J., Shizuya, H., de Jong, P.J., Birren, B., and Simon, M.I. 1992. Stable propagation of cosmid sized human DNA inserts in an $\mathrm{F}$ factor based vector. Nucleic Acids Res. 20: 1083-1085.

Kong, A., Gudbjartsson, D.F., Sainz, J., Jonsdottir, G.M., Gudjonsson, S.A., Richardsson, B., Sigurdardottir, S., Barnard, J., Hallbeck, B., Masson, G.A., et al. 2002. A high-resolution recombination map of the human genome. Nat. Genet. 31: 241-247.

Kotsch, K. and Blasczyk, R. 2000. The non-coding regions of HLA-DRB uncover inter-lineage recombinations as a mechanism of HLA diversification. In Major histocompatibility complex: Evolution, structure, and function (ed. M. Kasahara), pp. 412-426. Springer-Verlag, Tokyo.

Kwok, W.W., Kovats, S., Thurtle, P., and Nepom, G.T. 1993. HLA-DQ allelic polymorphisms constrain patterns of class II heterodimer formation. J. Immunol. 150: 2263-2272.

Lawlor, D.A., Ward, F.E., Ennis, P.D., Jackson, A.P., and Parham, P. 1988. HLA-A and B polymorphisms predate the divergence of humans and chimpanzees. Nature 335: $268-271$.

Lewontin, R.C. 1964. The interaction of selection and linkage. I. General considerations; heterotic models. Genetics 49: 49-67.

Marsh, S.G., Parham, P., and Barber, L.D. 2000. The HLA FactsBook. Academic Press, San Diego, CA.

Martin, M., Mann, D., and Carrington, M. 1995. Recombination rates across the HLA complex: Use of microsatellites as a rapid screen for recombinant chromosomes. Hum. Mol. Genet. 4: 423-428.

Mayer, W.E., Jonker, M., Klein, D., Ivanyi, P., van Seventer, G., and Klein, J. 1988. Nucleotide sequences of chimpanzee MHC class I alleles: Evidence for trans-species mode of evolution. $E M B O \mathrm{~J}$. 7: 2765-2774.

McGinnis, M.D., Lebo, R.V., Quinn, D.L., and Simons, M.J. 1994. Ancient, highly polymorphic human major histocompatibility complex DQA1 intron sequences. Am. J. Med. Genet. 52: 438444.

The MHC Sequencing Consortium 1999. Complete sequence and gene map of a human major histocompatibility complex. Nature 401: 921-923.

Price, P., Witt, C., Allcock, R., Sayer, D., Garlepp, M., Kok, C.C., French, M., Mallal, S., and Christiansen, F. 1999. The genetic basis for the association of the 8.1 ancestral haplotype (A1, B8, DR3) with multiple immunopathological diseases. Immunol. Rev. 167: 257274.

Sanchez-Mazas, A., Djoulah, S., Busson, M., Le Monnier de Gouville, I., Poirier, J.C., Dehay, C., Charron, D., Excoffier, L., Schneider, S. Langaney, A., et al. 2000. A linkage disequilibrium map of the MHC region based on the analysis of 14 loci haplotypes in 50 French families. Eur. J. Hum. Genet. 8: 33-41.

\section{Genome Research}

www.genome.org 


\section{Ancient haplotypes of the HLA Class II region}

Satta, Y. and Takahata, N. 2000. Polymorphism in the HLA class I region. In Major histocompatibility complex: Evolution, structure, and function (ed. M. Kasahara), pp. 412-426. Springer-Verlag, Tokyo.

Satta, Y., Kupfermann, H., Li, Y.-J., and Takahata, N. 1999. Molecular clock and recombination in primate Mhc genes. Immunol. Rev. 167: 367-379.

Shen, P. and Huang, H.V. 1989. Effect of base pair mismatches on recombination via the RecBCD pathway. Mol. Gen. Genet. 218: 358-360.

Smith, J.M. and Haigh, J. 1974. The hitch-hiking effect of a favourable gene. Genet. Res. 23: 23-35.

Stenzel, A., Lu, T., Koch, W.A., Hampe, J., Guenther, S.M., De La Vega, F.M., Krawczak, M., and Schreiber, S. 2004. Patterns of linkage disequilibrium in the MHC region on human chromosome $6 \mathrm{p}$. Hum. Genet. 114: 377-385.

Stewart, C.A., Horton, R., Allcock, R.J., Ashurst, J.L., Atrazhev, A.M Coggill, P., Dunham, I., Forbes, S., Halls, K., Howson, J.M. et al.
2004. Complete MHC haplotype sequencing for common disease gene mapping. Genome Res. 14: 1176-1187.

\section{Web site references}

http://www.phrap.org/; documentation and distribution information for phred, phrap, consed, and Autofinish.

http://www.anthonynolan.org.uk/HIG/data.html; curated compilation of known alleles of $D Q B 1, D Q A 1$, and $D R B 1$.

http://genome.ucsc.edu/; access to July, 2003 build of the human genome sequence, which was used as the source for RefSeq.

http://evolution.genetics.washington.edu/phylip.html; documentation and distribution information for Phylip.

Received December 10, 2004; accepted in revised form July 15, 2005. 


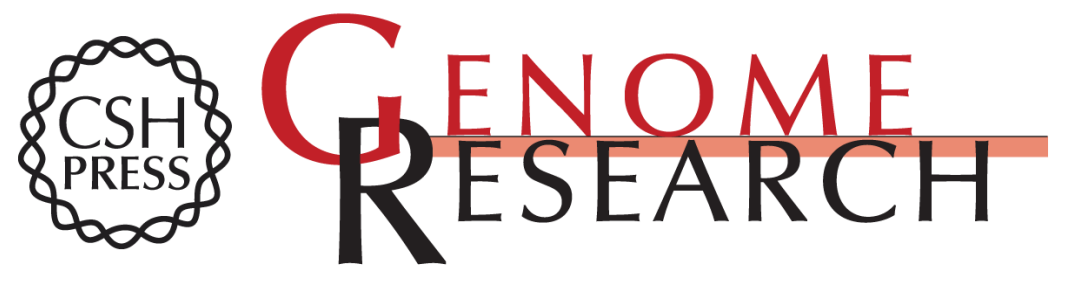

\section{Ancient haplotypes of the HLA Class II region}

Christopher K. Raymond, Arnold Kas, Marcia Paddock, et al.

Genome Res. 2005 15: 1250-1257

Access the most recent version at doi:10.1101/gr.3554305

\section{Supplemental http://genome.cshlp.org/content/suppl/2005/08/19/15.9.1250.DC1 Material}

References This article cites 41 articles, 14 of which can be accessed free at: http://genome.cshlp.org/content/15/9/1250.full.html\#ref-list-1

\section{License}

Email Alerting Receive free email alerts when new articles cite this article - sign up in the box at the Service top right corner of the article or click here.

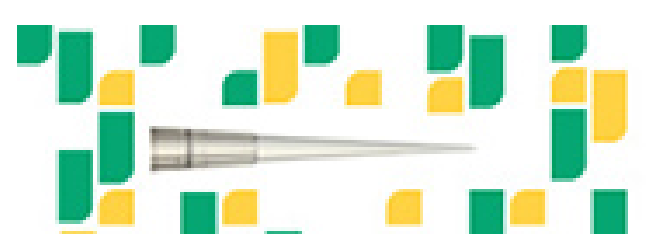

To subscribe to Genome Research go to: https://genome.cshlp.org/subscriptions 